\title{
Valoración de riesgo mediante modelos GARCH y simulación Montecarlo: evidencia del mercado accionario colombiano*
}

\author{
Recibido: 30 de noviembre de 2019 • Aprobado: 12 de diciembre de 2019 \\ https://doi.org/10.22395//seec.v22n53a3 \\ María Ines Barbosa Camargo ${ }^{* *}$ \\ Alejandra Salazar Sarmiento*** \\ Kelly Jhohana Peñaloza Gómez ${ }^{* * * *}$
}

\section{RESUMEN}

Este documento evalúa el comportamiento de varios modelos de volatilidad en estimaciones de un día del valor en riesgo (VaR) de veinticuatro series de retornos de acciones en Colombia con diferentes distribuciones. Al considerar que todas las series de retornos presentan clúster de volatilidad y memoria de largo plazo, se utilizan modelos tipo GARCH que incluyen diferentes distribuciones: normal, T-Student y GED. Los hallazgos corroboran la dificultad de elegir un único modelo para el cálculo del VaR, pero validan el uso de modelos paramétricos con distribución normal y simulación Montecarlo en mercados financieros emergentes.

\section{PALABRAS CLAVE}

Valor en riesgo; modelos GARCH; simulación; Colombia

\section{CLASIFICACIÓN JEL}

G17, G32, C58

\section{CONTENIDO}

Introducción; 1. Breve revisión de la literatura: valor en riesgo; 2. Metodología; 3. Resultados; Conclusiones; Bibliografía.

* El presente artículo es resultado de la investigación desarrollada en el marco del proyecto Modelación de los mercados financieros de Colombia, adscrito al Grupo de Investigación en Estudios Sociales, Financieros e Internacionales (ESFI), clasificado en categoría C en Colciencias (2018) y financiado por la Universidad de la Salle. Se realizó entre abril de 2018 y noviembre de 2019. El artículo se basado en el trabajo de grado obligatorio para optar al título de Profesional en Finanzas y Comercio Internacional de la Universidad de La Salle.

* Economista y magíster en Ciencias Económicas, Universidad Nacional de Colombia, Bogotá, Colombia Doctora en Análisis Económico Aplicado e Historia Económica, Universidad de Sevilla, Sevilla, España. Docente investigadora, Universidad de la Salle, Bogotá, Colombia. Integrante del Grupo de Investigación en Estudios Sociales, Financieros e Internacionales (ESFI), Bogotá, Colombia. Correo electrónico: mibarbosa@unisalle.edu.co. Orcid: https://orcid.org/0000-0002-7705-7983

** Profesional en Finanzas y Comercio Internacional, Universidad de la Salle, Bogotá, Colombia. Correo electrónico: salejandra64@unisalle.edu.co. Orcid: https://orcid.org/0000-0001-8445-5202

$\ldots$ Profesional en Finanzas y Comercio Internacional, Universidad de la Salle, Bogotá, Colombia. Correo electrónico: kpenaloza01@unisalle.edu.co. Orcid: http://orcid.org/0000-0001-6835-8920 


\title{
Value at Risk Through GARCH Models and Montecarlo Simulation: Evidence from the Colombian Stock Market
}

\begin{abstract}
This paper evaluates the performance of several volatility models for estimating one-day-ahead Value-at-Risk (VaR) of twenty-four stocks return series in Colombia, using a number of distributional assumptions. Because all return series exhibit volatility clustering and long-range memory, GARCH-type models including models under normal, T-Student and generalized error distribution are examined. The findings corroborate the difficulty of choosing a single model for calculating VaR, but validate the use of parametric models with normal distribution and Montecarlo simulation in emerging financial markets.
\end{abstract}

\section{KEYWORDS}

Value-at-Risk; GARCH-type models; simulation; Colombia.

\section{JEL CLASSIFICATION}

G17, G32, C58

\section{CONTENT}

Introduction; 1. Brief literature review; Value-at-Risk; 2. Methodology; 3. Results; Conclusions Bibliography.

\section{Avaliação do risco mediante modelos GARCH e simulação Montecarlo: evidência do mercado acionista colombiano}

\section{RESUMO}

Este documento avalia o comportamento de vários modelos de volatilidade em estimativas de um dia do Value at Risk (VaR) de 24 séries de retornos das ações na Colômbia com diferentes distribuições. Ao considerar que todas as séries de retornos apresentam cluster de volatilidade e memória de longo prazo, são utilizados modelos tipo GARCH que incluem diferentes distribuições: normal, t-student e GED. Os achados corroboram a dificuldade de escolher um único modelo para calcular o VaR, mas validam o uso de modelos paramétricos com distribuição normal e simulação Montecarlo em mercados financeiros emergentes.

\section{PALAVRAS-CHAVE}

Value at risk; modelos GARCH; simulação; Colômbia

\section{CLASSIFICAÇÃO JEL}

\section{G17, G32, C58}

\section{CONTEÚDO}

Introdução; 1. Breve revisão da literatura: value at risk; 2. Metodologia; 3. Resultados; Conclusões; Bibliografia. 


\section{INTRODUCCIÓN}

El mercado de capitales es un tipo de mercado financiero en el que se realiza compra y venta de títulos valor. Dicha compra y venta de títulos valor se realiza en contextos de incertidumbre bajo la conocida relación rendimiento-riesgo extendida por Markovitz (1952), establece que la inversión en activos más riesgosos se ve compensada con mayores rentabilidades esperadas. A partir de esta relación, se da lugar a diversas aproximaciones de la medición del riesgo de los activos, que incluyen medir la volatilidad mediante el uso de técnicas, tanto estadísticas como econométricas, que buscan realizar una mejor gestión del riesgo. Una de las medidas más ampliamente utilizadas es el Valor en Riesgo (VaR por sus siglas en inglés).

Según Fan et al., (2008), el Valor en Riesgo (VaR) se convierte en una herramienta esencial para los mercados financieros al ofrecer una medición del riesgo mediante el uso de una distribución de probabilidad de variable aleatoria. Incluso, el Comité de Supervisión Bancaria de Basilea (BCBS por sus siglas en inglés) (2019) propone dicha medida dentro del conjunto de medidas acordadas internacionalmente como respuesta a la crisis financiera de 2008, con el objetivo de reforzar la regulación, supervisión y gestión del riesgo de los bancos. Su cálculo se puede realizar mediante la elaboración de modelos econométricos que resultan muy útiles en la medición de riesgo. Al respecto, Su y Hung, (2011) y Su, Lee, y Chiu (2014) señalan que esto es deseable dado que permite incorporar las características de las series de tiempo financieras, tales como colas anchas, asimetría, clúster de volatilidad y memoria de largo plazo. De hecho, varios autores coinciden en que capturar el comportamiento asimétrico es esencial para un pronóstico más preciso del VaR. Por ejemplo, Wong, Chin y Tan (2016) llegan a este resultado a partir de la comparación de volatilidad de tres índices accionarios. Abounoori, Elmi y Nademi (2016) hallan que los modelos con mejor desempeño con un horizonte de tiempo de un día son AR(2)-MRSGARCH-GED. Por su parte, Cheong (2008) encuentra para Malasia que la distribución de Pareto es la que mejor recoge el comportamiento asimétrico de ambas colas.

En efecto, existen diversos trabajos que abordan de manera empírica la elaboración de modelos en la gestión de riesgo. Algunos de estos se centran en la incorporación de volatilidad a través de diferentes tipos de modelos GARCH, por ejemplo, Mabrouk (2016) utiliza modelos de largo plazo en siete índices accionarios y tres tipos de cambio; Schaeffer et al., (2012) realizan una medición de la volatilidad en acciones del sector petrolero; Laporta, Merlo y Petrella (2018) utilizan diferentes modelos GARCH y cuantiles para el pronóstico del VaR para siete commodities (materias primas o bienes primarios) de energía. 
Por su parte, trabajos como el de Youssef, Belkacem y Mokni (2015) confirman para el mercado del crudo y la gasolina que tener en cuenta elementos de memoria de largo plazo, asimetría y colas pesadas es importante para el manejo del riesgo y las estrategias de cobertura; Ewing y Malik (2017) resaltan la importancia de distinguir entre buenas y malas noticias e incorporar cambios estructurales en los modelos GARCH asimétricos para reducir la persistencia en volatilidad de los precios del crudo; Cheong (2009) utiliza modelos ARCH para estimar hechos estilizados de la volatilidad de los dos mayores mercados de crudo (WTI y Brent).

De igual manera, se encuentran estudios que utilizan modelos con aproximaciones diversas. Entre estos, aquellos implementados por Chiu y Chuang (2016) a las propiedades de memoria larga o persistencia en volatilidad de acciones; Bentes (2014) a los índices bursátiles; Tang y Shieh (2006) en tipos de cambio y mercados de futuros; Aloui y Mabrouk (2010) en commodities de crudo y gas; Degiannakis y Potamia (2017) para acciones, commodities y tipos de cambio. Asimismo, existen otros que diferencian entre posición corta y posición larga, por ejemplo, Giot y Laurent (2003) estiman un modelo de memoria larga basado en distribución T; So y Yu (2006) estiman siete modelos GARCH para doce índices de mercado y cuatro tasas de cambio, resaltan la importancia de las colas anchas en la estimación del VaR. Todo esto evidencia la amplia variedad de trabajos que incorporan en sus aplicaciones empíricas el uso de modelos econométricos para el cálculo del VaR.

Sin embargo, la mayoría de estas aproximaciones se realizaron en mercados financieros desarrollados, son pocos los estudios que se centran en la gestión del riesgo en mercados emergentes. Se resalta el trabajo de Yang y Chang (2008) que señala la importancia de evaluar el valor y la estabilidad de la moneda local como parte del análisis de decisión de inversión en el mercado accionario; el de Reboredo, Rivera-Castro y Ugolini (2016) que estructura la dependencia de la relación entre los retornos de acciones y los tipos de cambio como cobertura de un portafolio; aquellos que analizan los índices accionarios y los efectos en los mercados locales en economías emergentes como Brasil, Chile, Colombia, India, México, Rusia, Suráfrica y Turquía (Assaf, 2015; Dimitrakopoulos et al., 2010; Su, 2015; Su y Knowles, 2006; Diamandis et al., 2011; Sadorsky, 2014); así como el de Slim, Koubaa y BenSaida (2017) que encuentra que en el caso de mercados emergentes, el modelo GARCH estándar es la mejor especificación para los pronósticos de estimaciones VaR.

Particularmente para el caso colombiano, son pocos los trabajos que buscan presentar el comportamiento del mercado accionario, y aún más escasos los que hacen uso de modelos econométricos para la gestión del riesgo. De manera puntual, destacan los trabajos de Uribe (2007), quien realiza una comparación con índices de la 
región y ciertos mercados desarrollados, con el ánimo de fijar el grado de desarrollo local; Rivera (2009) analiza los patrones estacionales de los antiguos índices de las Bolsas de Bogotá (IBB), Medellín (Ibomed) y Colombia (IGBC) y encuentra evidencia que contradice la hipótesis de mercados eficientes; Pino, Uribe y Jiménez (2017) proponen una descripción del mercado de acciones, realizan un análisis de topología de red con el fin de reconocer la importancia institucional; y Delgado y Durango (2018) evalúan la estructuración de portafolios a partir de comparaciones con la volatilidad del índice Colcap que tiene en cuenta que este es el principal indicador bursátil del país, está compuesto por las acciones más transadas del mercado.

No obstante, a pesar de la escasa literatura, se hallaron tres artículos similares a nivel nacional: el primero lo realizaron Ospina y Giraldo (2009), quienes calcularon el VaR para las acciones de mayor bursatilidad del mercado colombiano mediante el método paramétrico, como resultado, encontraron que los modelos más significativos fueron los GARCH asimétricos. El segundo, desarrollado por Kuri, Ojeda y Ovalle (2015), estimó el VaR para la acción de Ecopetrol mediante modelos de heterocedastidad condicionada, encontraron que el tipo de estimación que mejor modela los retornos de esta acción es el GARCH $(1,1)$. El tercero -elaborado por Pérez y Fernández (2006) - considera la volatilidad del Índice General de la Bolsa de Valores de Colombia (IGBC).

En este contexto, esta investigación contribuye a la literatura de análisis de riesgo en mercados emergentes puesto que calcula el valor en riesgo (VaR) para cada una de las veinticuatro acciones de la canasta del índice Colcap que rige de mayo a julio de 2017, todo esto mediante la estimación de modelos econométricos que incorporan la volatilidad, asimetría y demás propiedades de las series financieras para el periodo comprendido entre agosto de 2013 y diciembre de 2017.

Asimismo, en mercados financieros emergentes como el colombiano, resulta de especial relevancia el análisis del riesgo de sus activos, teniendo en cuenta la vulnerabilidad que se presenta en pequeños mercados ante eventos extremos como las crisis financieras - de manera particular la crisis sub-prime de 2008 o la crisis hipotecaria de 1999-, así como las asimetrías de información que conllevan que los inversionistas realicen una toma de decisiones inadecuada.

En consecuencia, efectuar el cálculo del VaR para los activos propuestos y a partir de diversas metodologías, no solo permite identificar las principales características de las acciones del mercado de valores colombiano -y con ello aportar a la modelación de series financieras en mercados emergentes-, sino que también contribuye a situar la discusión de la gestión de riesgo frente a la importancia de 
contar con diversos métodos de análisis y formas de cálculo, en pos de una toma de decisiones más informada que conduzca a mejores rentabilidades y reducción del riesgo.

Este documento se estructura en cinco secciones que incluyen esta introducción. Posteriormente, se realiza una breve revisión de la literatura existente acerca del valor en riesgo, más adelante se describe la metodología utilizada para la elaboración de los modelos, el cálculo del VaR y el backtesting, seguido por una sección de resultados. La última sección presenta las conclusiones del artículo.

\section{BREVE REVISIÓN DE LITERATURA: VALOR EN RIESGO}

A menudo, la medición del riesgo de mercado se realiza a partir del VaR (valor en riesgo), el cual Jorion (2000, p. 13) define como "la peor pérdida esperada en un intervalo de tiempo determinado bajo condiciones normales del mercado ante un nivel de confianza dado". Al respecto, Sadegui y Shavvalpour (2006) describen el VaR como un método cuantitativo ampliamente adoptado por instituciones tales como los bancos, gestores de cartera y reguladores, al ser reconocida-según Dowd y Blake (2006) - como la medida más ampliamente utilizada en la práctica y cuyo cómputo resulta fundamental para el cálculo de otras medidas de riesgo basadas en cuantiles, como por ejemplo, el expected shortfall.

Existen diferentes formas de calcular el VaR, estas se pueden clasificar en paramétrica, no paramétrica y simulación Montecarlo. Según Hull y White (1998), el método paramétrico -también conocido como método analítico de la matriz de varianzas/ covarianzas- se basa en la distribución normal de los rendimientos de la cartera. El método no paramétrico se muestra independiente de cualquier distribución de probabilidad y por tal motivo, no se necesita estimar volatilidades ni correlaciones. El método de simulación Montecarlo permite la estimación de manera paramétrica y no paramétrica, ofrece una descripción más realista del riesgo a partir del ajuste de una distribución empírica de los retornos.

Según Cheong (2008), las estimaciones no paramétricas tienen las ventajas de la simplicidad y -lo que es más importante- la no necesidad de un supuesto de distribución específico para la serie de rendimientos, mientras que el cálculo del VaR basado en un modelo paramétrico requiere estimaciones precisas de la volatilidad y un cuantil correspondiente a la distribución empírica. En esta línea, Sadegui y Shavvalpour (2006) afirman que los estudios previos sobre la estimación del VaR basada en modelos se han centrado principalmente en la caracterización de los hechos estilizados que se encuentran con frecuencia en los datos financieros, 
especifican la estructura variable de la volatilidad y la forma de la distribución del rendimiento de los activos.

Dentro de la estimación paramétrica, el modelo más utilizado es el de RiskMetrics propuesto por J. P. Morgan, el cual asume que los rendimientos de los activos siguen una distribución normal con media cero y varianza condicional en un promedio móvil ponderado exponencialmente (EWMA) de los rendimientos históricos al cuadrado. Sin embargo, para González-Rivera, Lee y Mishra (2004), a pesar de su uso extendido, este modelo parece ser el que peor desempeño presenta. Lo anterior es consistente con el trabajo de Mabrouk y Saadi (2012) en cuanto que argumenta que dicha estimación del VaR es la menos precisa al no tener en cuenta las características más destacadas de las series de tiempo financieras: colas gruesas, asimetría, agrupamiento de volatilidad y memoria larga.

Además, es común que el primer supuesto (el de normalidad) no se presente en un contexto empírico, al tener en cuenta que las características de la distribución del retorno de activos involucran fenómenos de colas pesadas, leptocurtosis y asimetría. Por este motivo, el uso de una distribución condicional que no incluya dichas características puede llevar a un sesgo en la estimación del verdadero riesgo de mercado.

Por ello, estudios previos han hecho un gran esfuerzo para superar estos problemas al emplear conjuntos de distribuciones condicionales. Algunos de los trabajos pioneros son: Bollerslev (1987) con la T-Student, y Angelidis et al., (2004) con la distribución de errores generalizada (GED) de cola pesada, que permite capturar eventos extremos. Al respecto, Mabrouk (2016) añadió que ambas distribuciones son las más ampliamente utilizadas al presentar mejor desempeño al momento de capturar el exceso de curtosis. Sin embargo, las distribuciones GED y T-Student (que son simétricas) no pueden incluir el componente de asimetría presente en las innovaciones.

El segundo supuesto del promedio móvil ponderado exponencialmente para la varianza tampoco suele incorporar suficiente información con respecto a las propiedades empíricas de las dinámicas de volatilidad, como la memoria larga y el efecto de apalancamiento. Si bien para Hartz, Miitnik y Paolella (2006) existen diversos métodos que buscan estar en capacidad de incorporar las dos principales características dentro de los métodos que capturan estas dos características de una manera razonable -y que han sido ampliamente extendidas- se encuentran los modelos de heterocedasticidad condicionada, propuestos por Engle (1982) y generalizados por Bollerslev (1986). 
Dichos modelos han proporcionado una buena primera aproximación a estos hechos estilizados, al modelar la estructura autorregresiva en la varianza condicional. En efecto, trabajos como el de Yang y Chang (2008) evidencian que la estimación de la volatilidad en el rendimiento de los activos resulta crucial para la fijación de precios de las opciones, en la previsión de umbrales de valor en riesgo (VaR) y en la selección óptima de la cartera, al encontrar que los modelos GARCH son adecuados para describir datos como clúster de volatilidad y comportamiento de cola gruesa.

Sin embargo, de acuerdo con autores como Andersen et al., (2002) y Gallant et al., (1997), los modelos tipo GARCH presentan alta persistencia y suelen no ser los más adecuados frente a eventos raros en retornos de activos. Estos saltos pueden llevar a una sobreestimación del riesgo de mercado real. De allí que se hayan desarrollado modelos que incorporan propiedades adicionales, como la asimetría, la cual se refiere a los impactos diferenciales de choques positivos y negativos de igual magnitud en la volatilidad. Según Cheong (2008), esta propiedad es muy importante en el análisis de riesgo en el cual las inversiones en posiciones largas y cortas durante un período de tiempo determinado se basan en gran medida en los comportamientos de las colas inferiores y superiores.

Para incorporar esta propiedad, Nelson (1991) desarrolló el modelo exponencial GARCH, EGARCH, que analiza los efectos positivos y negativos ocasionados por las noticias del mercado de valores, con choques negativos que afectan la volatilidad de manera diferente a los choques positivos de igual magnitud. Además, la posibilidad de apalancamiento que permite que los choques negativos aumenten la volatilidad — mientras que los choques positivos disminuyen la volatilidad — está presente en la formulación de los modelos EGARCH. Bajo la misma idea, se han desarrollado modelos asimétricos alternativos de volatilidad, como el umbral GARCH, TGARCH, de Zakoian (1994). Al respecto, Yang y Chang (2008) y Mabrouk (2016) señalan que tales modelos típicamente distinguen entre la asimetría de información, un umbral, y umbrales de valores críticos.

Consecuentemente, validaciones empíricas como la desarrollada por Su y Hung (2011) que resaltan, tanto las colas pesadas en el cálculo del VaR al $99 \%$ en posiciones largas, como la asimetría y choques dinámicos, así como la de Bayer (2018), que diferencia entre ventanas y el uso de técnicas que combinan varios métodos, han mostrado mejoras en la eficiencia.

Por otra parte, la evaluación del pronóstico mayormente generalizada es aquella realizada a partir de backtesting, que el BCBS (2019) define como el proceso utilizado para comparar las ganancias y pérdidas diarias reales e hipotéticas, con las medidas 
de VaR generadas por el modelo para evaluar el conservadurismo de los sistemas de medición de riesgos.

La aproximación más utilizada es la propuesta por Kupiec (1995), también conocida como la versión incondicional de la prueba de cobertura, la cual se centra en si el número real de excepciones de VaR es igual al número esperado de excepciones, es decir, examina si la tasa de cobertura realizada (ex-post) es igual a la tasa de cobertura teórica. De acuerdo con Dimitrakopoulos et al., (2010) y Laporta et al., (2018), dicha prueba sigue un proceso de Bernoulli con parámetro p en la cual p es igual a la tasa teórica de cobertura del VaR, de esta manera, la variable toma el valor de 1 si la pérdida excede el VaR estimado y 0 en los otros casos.

Por definición, la tasa de fallas supone el número de veces que el retorno excede -en valor absoluto- el pronóstico del VaR. Si el modelo VaR se especifica correctamente, la tasa de fallas debería ser igual al nivel VaR especificado. La tasa de fallas empíricas $\mathrm{f}$ se define como una secuencia de observaciones sí/no, de tal manera que es posible probar si el modelo se especifica de manera adecuada. A un nivel del $95 \%$, un intervalo de confianza para la tasa de fallas se puede construir por:

$$
\hat{f} \pm 1.96 \sqrt{\hat{f}(1-\hat{f}) / T}
$$

El estadístico LR es

$$
L R=-2 \log \left(\frac{\alpha^{\mathrm{N}}(1-\alpha)^{\mathrm{T}-\mathrm{N}}}{\hat{f}^{\mathrm{N}}(1-\hat{f})^{\mathrm{T}-\mathrm{N}}}\right)
$$

donde $\mathrm{N}$ es el número de violaciones del VaR, T es el número total de observaciones y $\alpha$ es la tasa de fallas teóricas. Bajo la hipótesis nula de que la tasa de fallas es correcta, el estadístico LR se distribuye como un chi-cuadrado con un grado de libertad.

\section{METODOLOGÍA}

\subsection{Datos}

El análisis de riesgo realizado para el mercado accionario colombiano utiliza para el cálculo del VaR las principales acciones que componen el índice Colcap, en este índice bursátil participan las acciones más liquidas que cotizan en la Bolsa de Valores de Colombia, el cual debe estar compuesto por mínimo veinte acciones de emisores diferentes. 
El periodo de análisis comprende desde el $1^{\circ}$ de agosto de 2013 hasta el 28 de diciembre de 2017, con un total de 1.074 observaciones para cada una de las 24 acciones que conforman la canasta del índice Colcap de mayo a julio de 2017. El periodo de estimación elegido busca tener la misma cantidad de observaciones por acción, de allí que considere el hecho de que la acción de Avianca Holdings cambió su nemotécnico el 19 de julio de 2013 de PFAVTA a PFAVH.

Los precios de cierre diarios de las acciones se obtienen de la Bolsa de Valores de Colombia (BVC). Los retornos diarios se calculan como la diferencia logarítmica en el precio de cierre diario, es decir, $\mathrm{R}_{t}=\log \left(\mathrm{P}_{r}\right)-\log \left(\mathrm{P}_{r-1}\right)$ donde $\mathrm{P}_{t}$ es el precio de cierre diario. La tabla 1 describe las empresas que componen el índice Colcap para el periodo de análisis.

\section{Tabla 1. Empresas}

\begin{tabular}{ll} 
Nemotécnico & \multicolumn{1}{c}{ Descripción empresa } \\
\hline BCOLOMBIA, & $\begin{array}{l}\text { Bancolombia es una organización financiera colombiana; es el banco privado } \\
\text { más grande del país (por el tamaño de su patrimonio y activos) y uno de los más } \\
\text { PFBCOLOM }\end{array}$ \\
\hline
\end{tabular}

Ecopetrol S.A., es la empresa más grande del país y la principal compañía ECOPETROL petrolera en Colombia. Por su tamaño, es una de las cinco principales petroleras de Latinoamérica.

EXITO Éxito es una compañía multilatina, líder del mercado al detal en Suramérica. Presentes en Colombia con el Grupo Éxito.

\section{ETB}

La Empresa de Telecomunicaciones de Bogotá (ETB), cuenta con un gran potencial, gracias a sus servicios de fibra óptica, televisión interactiva digital y móviles 4G.
La Bolsa de Valores de Colombia (BVC) es una bolsa multi-producto, multi-mercado integrada verticalmente $Y$ que administra las plataformas de negociación y registro para los mercados de acciones, renta fija, derivados, divisas, OTC y servicios a emisores. BVC
CONCONCRET
Conconcreto es una compañía líder en el desarrollo de proyectos de infraestructura y edificación en Colombia y la región, con más de 55 años en el mercado.

CNEC

Canacol Energy es una compañía líder en la exploración y producción de petróleo y gas. Se estableció como corporación privada en 2008. Sus operaciones están concentradas en Colombia y Ecuador.

BOGOTA

El Banco de Bogotá es la primera institución financiera creada en el país, cubre la totalidad del territorio nacional y continúa expandiendo sus operaciones internacionales con sus filiales y agencias en el exterior. 
Corficolombiana es una corporación financiera controlada por Grupo Aval; enfocada

CORFICOLCF en tres unidades de negocio principales: inversiones en portafolio, tesorería y banca de inversión.

PFDAVVNDA

Davivienda es un banco que hace parte del Grupo Empresarial Bolívar, es el banco exclusivo en Colombia para el manejo de las tarjetas Diners.

El Grupo Energía Bogotá (GEB) es una multilatina líder en el sector de energía

EEB eléctrica y gas natural. Su estrategia está centrada en tres grupos estratégicos de negocio: soluciones energéticas urbanas, interconexión para el desarrollo de mercados y generación de baja emisión.

El Grupo Empresarial SURA es una organización multilatina co GRUPOSURA, de experiencia y con inversiones estratégicas en los sectores de banca, seguros, PFGRUPSURA. pensiones, ahorro e inversión; a través de las cuales entrega soluciones financieras integrales.

Interconexión Eléctrica S. A., es una organización que focaliza sus actividades en ISA los negocios de transporte de energía eléctrica, concesiones viales, tecnologías de información y telecomunicaciones, y gestión de sistemas de tiempo real.

En el negocio del cemento, Argos es líder en Colombia, quinto productor más grande

CEMARGOS, en América Latina y segundo más grande en el sureste de Estados Unidos. Por otro PFCEMARGOS lado, en el negocio del concreto es líder en Colombia y es el tercer productor más grande en Estados Unidos.

Es la empresa de energía del Grupo Argos, tiene presencia en Colombia, Panamá

CELSIA y Costa Rica, con una capacidad de generación de 2.399 MW desde 28 centrales hidroeléctricas, térmicas, fotovoltaicas y eólicas que generan alrededor de 7.750 GWh anuales.

Grupo Nutresa S.A. es la empresa líder en alimentos procesados en Colombia. Opera NUTRESA a través de ocho unidades de negocio: cárnicos, galletas, chocolates, Tresmontes Lucchetti (TMLUC), cafés, alimentos al consumidor, helados y pastas.

GRUPOARGOS, PFGRUPOARG

Grupo Argos es una matriz de inversiones sostenibles en infraestructura, en compañías listadas en bolsa y empresas privadas. Su naturaleza jurídica corresponde a una sociedad anónima.

Grupo Aval es el conglomerado financiero más grande de Colombia, su portafolio

PFAVAL cuenta con una corporación financiera y con negocios de administración de fondos de pensiones y cesantías.

Cemex Latam Holdings es una compañía de materiales para la industria de la $\mathrm{CLH}$ construcción que ofrece productos de alta calidad a clientes y comunidades en Colombia.

Avianca Holdings S.A. es una compañía latinoamericana que tiene inversiones PFAVH principalmente en: aerolíneas de pasajeros, aerolíneas de carga y actividades relacionadas.

Fuente: elaboración propia a partir de las páginas oficiales de las empresas. 


\subsection{Estimación de modelos}

Como se describió en la revisión de literatura, una mejor aproximación al riesgo debe incluir la estimación de modelos que incluyan características de las series financieras como clúster de volatilidad, asimetría y apalancamiento. Debido a la naturaleza de las series financieras presente en los retornos diarios, la estimación de los modelos contempló dos fases. En primer lugar, la definición de una ecuación para la media, habitualmente estimada mediante modelos autorregresivos de media móvil; en segundo lugar, la estimación de la varianza condicionada, la cual se puede dar mediante modelos de heterocedasticidad condicionada GARCH, E-GARCH y TARCH.

Para la estimación de dichos modelos se tuvo en cuenta que las series de retornos no se distribuyen de manera normal, por lo que se hizo uso de las distribuciones condicionales mayormente utilizadas, de acuerdo con lo propuesto en la literatura: la distribución T-Student y la distribución del error generalizado (GED). La primera es leptocúrtica, lo que significa que la parte central de la distribución es más delgada y tiene colas más anchas que la distribución normal, mientras que la segunda se extiende exponencialmente en la cola de la distribución (Castaño, Gómez y Gallón, 2008; Mendoza y Galvanovskis, 2014).

\subsection{Cálculo del VaR}

El cálculo del VaR se realizó bajo tres metodologías: no paramétrico, paramétrico y simulación Montecarlo. Las estimaciones se efectuadron con los softwares E-views ${ }^{\circledast}$, y Risk Simulator ${ }^{\circledast}$, respectivamente. Como su nombre lo indica, la simulación Montecarlo crea diversos modelos de posibilidades al calcular una y otra vez resultados (10.000 simulaciones) que comparan cada vez un grupo diferente de valores aleatorios de las funciones de probabilidad utilizadas y selecciona el mejor modelo de acuerdo con medidas de bondad de ajuste.

Una vez se obtuvieron los modelos para cada una de las series de retornos de los activos seleccionados, se continuó con el cálculo del VaR paramétrico a partir de la fórmula:

$$
\operatorname{VaR}_{\alpha}=\mu_{i, t}-z_{i, \alpha} \sqrt{h_{i, t}}
$$

Donde $\mu_{i, t}$ es el retorno condicional esperado del activo i, $\mathrm{Z}_{m, \alpha}$ corresponde al cuantil izquierdo $\alpha$ de la distribución usada en la serie de los residuales del modelo estimado en cada caso, y $h_{i, t}$ es la varianza condicional del activo $i$. Todos los cálculos se hicieron con un nivel de confianza del 95 \% lo que según Jorion (2000), indica 
que el VaR se encuentra en el $5 \%$ del lado izquierdo de la cola del histograma de cada una de las series.

Para determinar las acciones más riesgosas, cada medida de riesgo VaR se compara de acuerdo con la metodología empleada, a partir de la prueba de backtesting realizada, con el fin de validar la eficacia de los modelos implementados para la gestión de riesgo.

\section{RESULTADOS}

La tabla 2 presenta las ecuaciones de los modelos estimados para cada uno de los retornos de los precios de cierre de las 24 acciones que componen el índice Colcap. Algunos de estos retornos no requirieron ecuación para la media $(\mathrm{Rt}=0)$, pero todos incorporaron componentes de volatilidad bajo la especificación de modelos GARCH con distribuciones no-normales o con propiedades de asimetría.

Tabla 2. Modelos estimados

\begin{tabular}{|c|c|}
\hline Acción & Modelo \\
\hline $\begin{array}{l}\text { BCOLOMBIA AR } \\
(32)-\text { GARCH }(3,3) \sim \text { GED }\end{array}$ & $\begin{aligned} R_{t}= & -0.047876 * R_{t-32}+\varepsilon_{t} \\
\sigma_{t}^{2}= & 0.0000164+0.0939 * \varepsilon_{t-1}^{2}+0.0695 * \varepsilon_{t-2}^{2}+0.0541 * \varepsilon_{t-3}^{2}+0.0899 \\
& * \sigma_{t-1}^{2}-0.2640 * \sigma_{t-2}^{2}+0.8945 * \sigma_{t-3}^{2}\end{aligned}$ \\
\hline $\begin{array}{l}\text { PFBCOLOMBIA } \\
\text { MA (27)-GARCH }(4,5) \sim \\
\text { T-Student }\end{array}$ & $\begin{aligned} R_{t}= & 0.054354 * n_{t-27}+\varepsilon_{t} \\
\sigma_{t}^{2}= & 0.000031+0.1682 * \varepsilon_{t-1}^{2}+0.1132 * \varepsilon_{t-4}^{2}+0.4300 \\
& * \sigma_{t-2}^{2}-0.7177 * \sigma_{t-3}^{2}+0.5617 * \sigma_{t-5}^{2}\end{aligned}$ \\
\hline $\begin{array}{l}\text { ECOPETROL } \\
\text { AR (31) -GARCH }(6,7) \sim \\
\text { T-Student }\end{array}$ & $\begin{aligned} R_{t}= & 0.114729 * \varepsilon_{t-31}+\varepsilon_{t} \\
\sigma_{t}^{2}= & 0.1388 * \varepsilon_{t-1}^{2}+0.0750 * \varepsilon_{t-2}^{2}+0.0755 * \varepsilon_{t-3}^{2}+0.0945 * \varepsilon_{t-4}^{2}+0.0682 \\
& * \varepsilon_{t-5}^{2}+0.1181 * \varepsilon_{t-6}^{2}-0.2889 * \sigma_{t-1}^{2}+0.1276^{2} \sigma_{t-2}^{2}-0.3375 * \sigma_{t-3}^{2} \\
& +0.1568 * \sigma_{t-4}^{2}-0.2912 * \sigma_{t-5}^{2}+0.2252 * \sigma_{t-6}^{2}+0.8346 * \sigma_{t-7}^{2}\end{aligned}$ \\
\hline $\begin{array}{l}\text { EXITO } \\
\text { ARMA }(25,30)-G A R C H \\
(1,1) \sim \text { T-Student }\end{array}$ & $\begin{array}{l}R_{t}=-0.0449299 * R_{t-25}+0.048832 * \varepsilon_{t-28}+0.062261 * \varepsilon_{t-30}+\varepsilon_{t} \\
\sigma_{t}^{2}=0.0000026+0.1792 * \varepsilon_{t-1}^{2}+0.8430 * \sigma_{t-1}^{2}\end{array}$ \\
\hline $\begin{array}{l}\text { ETB } \\
\text { ARMA }(35,1)-G A R C H \\
(1,1) \sim \text { T-Student }\end{array}$ & $\begin{array}{l}R_{t}=-0.207126 * D 1-0.043069 * R_{t-35}-0.066520 * \varepsilon_{t-1}+\varepsilon_{t} \\
\sigma_{t}^{2}=0.000404+2.6314 * \varepsilon_{t-1}^{2}+0.5379 * \sigma_{t-1}^{2}\end{array}$ \\
\hline $\begin{array}{l}\text { BVC } \\
\text { AR (5)-GARCH }(2,2) \sim \\
\text { T-Student }\end{array}$ & $\begin{array}{l}R_{t}=-0.090457 * R_{t-1}-0.054099 * R_{t-5}+\varepsilon_{t} \\
\sigma_{t}^{2}=0.5143 * \varepsilon_{t-1}^{2}-0.4690 * \varepsilon_{t-2}^{2}+1.4144 * \sigma_{t-1}^{2}-0.4374 * \sigma_{t-2}^{2}\end{array}$ \\
\hline
\end{tabular}


María Ines Barbosa Camargo, Alejandra Salazar Sarmiento y Kelly Jhohana Peñaloza Gómez

Acción

Modelo

$R_{t}=0$

$\sigma^{2}{ }_{t}=0.000078+0.1642 * \varepsilon^{2}{ }_{t-1}-0.0301 * \varepsilon^{2}{ }_{t-1} d_{t-1}-0.0761 * \varepsilon^{2}{ }_{t-2}+0.0312$

CONCONCRET $* \varepsilon_{t-3}^{2}+0.0549 * \varepsilon_{t-4}^{2}+0.3893 * \sigma_{t-1}^{2}$;

TGARCH $(4,1) \sim \operatorname{GED}$

$d_{t-1}=\left\{\begin{array}{ccc}1 & \text { si } & \varepsilon_{t-1}<0 \\ 0 & \text { en otro caso }\end{array}\right.$

CNEC

$R_{t}=0$

GARCH $(1,1) \sim$ GED

$\sigma_{t}^{2}=0.0566 * \varepsilon^{2}{ }_{t-1}+0.9414 * \sigma_{t-1}^{2}$

BOGOTA

$R_{t}=-0.156928 * \varepsilon_{t-1}+\varepsilon_{t}$

MA(1)-E-GARCH $(1,1)$

T-Student

$\ln \left(\sigma^{2}{ }_{t}\right)=-1.229772+0.846173 *\left|\frac{\varepsilon_{t-1}}{\sigma_{t-1}}\right|+0.871493 * \ln \left(\sigma_{t-1}^{2}\right)$

\section{CORFICOLCF}

AR (1)-GARCH $(1,1)$

T-Student

PFDAVVNDA ARMA $(23,7)-\operatorname{GARCH}(1,1)$

T-Student

\section{EEB}

ARMA $(2,27)-G A R C H$

$(1,1) \sim$ T-Student

GRUPOSURA

GARCH $(4,4) \sim$

T-Student
$R_{t}=-0.078657 * R_{t-1}+\varepsilon_{t}$

$\sigma^{2}{ }_{t}=0.0000153+0.2295 * \varepsilon_{t-1}^{2}+0.7060 * \sigma_{t-1}^{2}$

$R_{t}=-0.709601 * R_{t-5}-0.035773 * R_{t-23}+0.720247 * \varepsilon_{t-5}-0.044815 * \varepsilon_{t-7}+\varepsilon_{t}$

$\sigma^{2}{ }_{t}=0.00000717+0.1028 * \varepsilon^{2}{ }_{t-1}+0.8705 * \sigma_{t-1}^{2}$

$R_{t}=-0.120129 * R_{t-1}-0.095815 * R_{t-2}+0.085742 * \varepsilon_{t-27}+0.048189 * \varepsilon_{t-28}+\varepsilon_{t}$

$\sigma_{t}^{2}=0.0000304+0.3613 * \varepsilon_{t-1}^{2}+0.5791 * \sigma_{t-1}^{2}$

$R_{t}=0$

$\sigma^{2}{ }_{t}=0.1999 * \varepsilon_{t-1}^{2}-0.1456 * \varepsilon_{t-2}^{2}+0.1511 * \varepsilon_{t-3}^{2}-0.1878 * \varepsilon_{t-4}^{2}$

$+1.4324 * \sigma_{t-1}^{2}-1.2996 * \sigma_{t-2}^{2}+1.5026 * \sigma_{t-3}^{2}-0.6537 * \sigma_{t-4}^{2}$

PFGRUPSURA GARCH $R_{t}=0$

$(3,1) \sim$ GED

$\sigma^{2}{ }_{t}=0.2620 * \varepsilon_{t-1}^{2}-0.1050 * \varepsilon_{t-2}^{2}-0.1155 * \varepsilon_{t-3}^{2}+0.9578 * \sigma_{t-1}^{2}$

ISA

ARMA $(31,8)-G A R C H$

$R_{t}=-0.083765 * R_{t-3}-0.050359 * R_{t-31}+0.066752 * \varepsilon_{t-8}+\varepsilon_{t}$

$(1,1) \sim$ T-Student

$\sigma_{t}^{2}=0.00000512+0.1268 * \varepsilon_{t-1}^{2}+0.8574 * \sigma_{t-1}^{2}$

CEMARGOS

MA(1)-GARCH $(1,1) \sim$

T-Student

PFCEMARGOS ARMA

$(23,10)-\operatorname{GARCH}(1,1)$

T-Student
$R_{t}=-0.104848 \varepsilon_{t-1}+\varepsilon_{t}$

$\sigma_{t}^{2}=0.0000051+0.1062 * \varepsilon_{t-1}^{2}+0.8817 * \sigma_{t-1}^{2}$

$R_{t}=-0.079853 * R_{t-22}-0.077593 * R_{t-23}+0.086457 * \varepsilon_{t-10}+\varepsilon_{t}$

$\sigma^{2}{ }_{t}=0.0000103+0.1118 * \varepsilon_{t-1}^{2}+0.8473 * \sigma_{t-1}^{2}$ 
Valoración de riesgo mediante modelos GARCH y simulación Montecarlo: evidencia del mercado accionario colombiano

Acción

Modelo

CELSIA

AR(1)-GARCH $(1,1)$

T-Student

NUTRESA

GARCH $(5,3) \sim \operatorname{GED}$

$$
\begin{aligned}
& R_{t}=-0.057508 * R_{t-1}+\varepsilon_{t} \\
& \sigma^{2}{ }_{t}=0.00000261+0.0960 * \varepsilon_{t-1}^{2}+0.9037 * \sigma^{2}{ }_{t-1}
\end{aligned}
$$$$
R_{t}=0
$$$$
\sigma^{2}{ }_{t}=0.0000122+0.1646 * \varepsilon_{t-1}^{2}+0.2200 * \varepsilon_{t-5}^{2}+0.4587 * \sigma_{t-3}^{2}
$$

GRUPOARGOS ARMA $(4,30)$-GARCH $(1,1)$

T-Student

$$
\begin{aligned}
& R_{t}=-0.069300 * R_{t-3}-0.072500 * R_{t-4}+0.055745 * \varepsilon_{t-30}+\varepsilon_{t} \\
& \sigma^{2} t=0.00000525+0.1254 * \varepsilon_{t-1}^{2}+0.8638 * \sigma_{t-1}^{2}
\end{aligned}
$$

PFGRUPOARG ARMA

$(3,30)-$ GARCH $(1,1)$

T-Student

$R_{t}=-0.122603 * R_{t-3}+0.074404 * \varepsilon_{t-9}+0.064724 * \varepsilon_{t-24}+0.055038 * \varepsilon_{t-30}+\varepsilon_{t}$

$\sigma_{t}^{2}=0.00000768+0.1755 * \varepsilon_{t-1}^{2}+0.8122 * \sigma_{t-1}^{2}$

PFAVAL

$$
R_{t}=0
$$

GARCH $(4,5) \sim$

$\sigma_{t}^{2}=0.0000271+0.2133 * \varepsilon_{t-1}^{2}+0.1731 * \varepsilon_{t-4}^{2}+0.1358 * \sigma_{t-1}^{2}+0.2005$

T-Student

$$
* \sigma_{t-2}^{2}-0.5122 * \sigma_{t-3}^{2}-0.1141 * \sigma_{t-4}^{2}+0.6899 * \sigma_{t-5}^{2}
$$

$$
R_{t}=0
$$

CLH

$\mathrm{ARCH}(6) \sim \mathrm{GED}$

$$
\begin{aligned}
\sigma^{2}= & 0.000113+0.1662 * \varepsilon_{t-1}^{2}+0.1827 * \varepsilon_{t-2}^{2}+0.0826 \\
& * \varepsilon_{t-3}^{2}+0.1906 * \varepsilon_{t-4}^{2}+0.0441 * \varepsilon_{t-5}^{2}+0.0738 * \varepsilon_{t-6}^{2}
\end{aligned}
$$

\section{PFAVH}

$\operatorname{GARCH}(2,1)$

T-Student

$$
R_{t}=0
$$$$
\sigma_{t}^{2}=0.0000114+0.3200 * \varepsilon_{t-1}^{2}-0.2705 * \varepsilon_{t-2}^{2}+0.9342 * \sigma_{t-1}^{2}
$$

\section{Fuente: elaboración propia}

La acción CLH presenta uno de los modelos más sencillos de los veinticuatro estimados, el cual es el ARCH. Por otra parte, veintiún modelos estimados corresponden al GARCH que es el modelo más usado para modelar series financieras. Asimismo, se puede evidenciar que la mayoría de los modelos son GARCH (1,1), lo que concuerda con los hallazgos de Slim, Koubaa y BenSaida (2017), quienes señalan para el caso de mercados emergentes que, el modelo GARCH estándar es la mejor especificación para los pronósticos de estimaciones VaR.

Las dos acciones restantes, que corresponden a Conconcreto y al Banco de Bogotá, requieren la estimación de modelos E-GARCH y TARCH respectivamente, los cuales corrigen los problemas que presentan los GARCH al incorporar el componente de asimetría. Lo anterior coincide con la importancia de estimar modelos que incluyan características propias de las series financieras y está ampliamente expuesto en la literatura (So y Yu, 2006; Yang y Chang, 2008; Youssef et al., 2015). 
María Ines Barbosa Camargo, Alejandra Salazar Sarmiento y Kelly Jhohana Peñaloza Gómez

De otra parte, los resultados del cálculo del VaR se presentan en la tabla 3. Es de anotar que el método paramétrico implementado utiliza tanto las distribuciones de los modelos de heterocedasticidad condicionada estimados, como la normal y la simulación Montecarlo.

Tabla 3. Comparación del VaR

\begin{tabular}{|c|c|c|c|c|c|c|}
\hline \multirow{3}{*}{ Acciones } & \multicolumn{5}{|c|}{ VaR paramétrico } & \multirow{3}{*}{$\begin{array}{c}\text { VaR no } \\
\text { paramétrico } \\
(\%)\end{array}$} \\
\hline & \multicolumn{3}{|c|}{ Modelos de heteroscedasticidad condicionada } & \multirow{2}{*}{$\begin{array}{c}\text { Distribución } \\
\text { normal (\%) }\end{array}$} & \multirow{2}{*}{$\begin{array}{l}\text { Simulación de } \\
\text { Montecarlo (\%) }\end{array}$} & \\
\hline & Media (\%) & Varianza (\%) & Modelos (\%) & & & \\
\hline BCOLOMBIA & 0,010 & 0,013 & $-1,88$ & $-2,42$ & $-2,46$ & $-2,46$ \\
\hline PFBCOLOMBIA & 0,038 & 0,0196 & $-2,27$ & $-2,30$ & $-2,63$ & $-2,17$ \\
\hline ECOPETROL & $-0,298$ & 0,057 & $-4,24$ & $-3,38$ & $-3,37$ & $-3,22$ \\
\hline EXITO & $-0,005$ & 0,003 & $-0,93$ & $-2,87$ & $-2,84$ & $-3,05$ \\
\hline ETB & 0,077 & 0,170 & $-6,71$ & $-2,49$ & $-2,57$ & $-2,02$ \\
\hline BVC & $-0,013$ & 0,041 & $-3,35$ & $-2,41$ & $-2,34$ & $-2,48$ \\
\hline CONCONCRET & 0,000 & 0,045 & $-3,48$ & $-2,42$ & $-3,08$ & $-2,15$ \\
\hline CNEC & 0,000 & 0,023 & $-2,49$ & $-5,41$ & $-5,19$ & $-4,62$ \\
\hline BOGOTA & 0,163 & 0,060 & $-3,86$ & $-2,03$ & $-1,29$ & $-1,98$ \\
\hline CORFICOLCF & $-0,038$ & 0,008 & $-1,52$ & $-2,09$ & $-1,94$ & $-1,99$ \\
\hline PFDAVVNDA & $-0,022$ & 0,009 & $-1,62$ & $-2,38$ & $-2,35$ & $-2,36$ \\
\hline EEB & 0,056 & 0,011 & $-1,67$ & $-2,04$ & $-1,12$ & $-1,90$ \\
\hline GRUPOSURA & 0,000 & 0,007 & $-1,42$ & $-2,16$ & $-2,16$ & $-2,29$ \\
\hline PFGRUPSURA & 0,000 & 0,013 & $-1,89$ & $-2,14$ & $-2,31$ & $-2,38$ \\
\hline ISA & 0,019 & 0,011 & $-1,68$ & $-2,46$ & $-2,48$ & $-2,40$ \\
\hline CEMARGOS & $-0,018$ & 0,013 & $-1,91$ & $-2,59$ & $-2,61$ & $-2,62$ \\
\hline PFCEMARGOS & 1,898 & 0,028 & $-0,86$ & $-2,15$ & $-2,18$ & $-2,12$ \\
\hline CELSIA & 0,048 & 0,012 & $-1,74$ & $-2,48$ & $-2,53$ & $-2,34$ \\
\hline NUTRESA & 0,000 & 0,133 & $-5,99$ & $-1,72$ & $-1,75$ & $-1,70$ \\
\hline GRUPOARGOS & 0,126 & 0,009 & $-1,44$ & $-2,63$ & $-2,63$ & $-2,75$ \\
\hline PFGRUPOARG & 0,280 & 0,013 & $-1,63$ & $-2,66$ & $-2,68$ & $-2,73$ \\
\hline PFAVAL & 0,000 & 0,019 & $-2,25$ & $-1,89$ & $-1,89$ & $-1,76$ \\
\hline $\mathrm{CLH}$ & 0,000 & 0,262 & $-8,42$ & $-2,95$ & $-2,99$ & $-0,82$ \\
\hline PFAVH & 0,000 & 0,025 & $-2,60$ & $-3,66$ & $-2,94$ & $-3,04$ \\
\hline
\end{tabular}

Fuente: elaboración propia. 
Del cálculo del VaR tradicional que hace uso de la distribución normal se resalta que las acciones que presentan mayor riesgo son: CNEC (5,41 \%), PFAV (3,66 \%) y ECOPETROL (3,38\%). En contraste, con el VaR paramétrico de los modelos de heteroscedasticidad condicionada estimados -los cuales asumen distribuciones T-Student o GED- las acciones más riesgosas son: CLH (8,42 \%), ETB (6,71 \%) y NUTRESA (5,99\%). Por su parte, la tercera metodología paramétrica o simulación Montecarlo que asumió otras distribuciones como: Laplace, Gumbel mínimo, logística, parabólico y normal, evidenció como acciones más riesgosas a: CNEC (5,19 \%), ECOPETROL (3,37 \%) y CONCONCRET (3,08\%). Por último, según el VaR no paramétrico las acciones más riesgosas son: CNEC (4,62 \%), ECOPETROL (3,22 \%) y EXITO (3,05\%).

Si bien, hay diferencias en los riesgos evidenciados a partir del cálculo del VaR con las metodologías utilizadas, acciones como ECOPETROL y CNEC resultan las más riesgosas en tres de las cuatro metodologías utilizadas, lo que sugiere que habría que tratarse con cuidado su incorporación en decisiones de inversión. Por el contrario, acciones como NUTRESA o EEB se consideran menos riesgosas que las demás y por tanto, más atractivas para la conformación de carteras.

Para verificar cual metodología se ajusta mejor, se emplea la probabilidad arrojada por el backtesting, la cual nace a partir de: P en días, que es el número de fallas que soporta el modelo en días; M no P, que son las fallas que presentan cada una de las metodologías; y PROB, que es la probabilidad, la cual no puede ser inferior al $5 \%$, debido a que se tomó el $95 \%$ como nivel de confianza para realizar esta prueba (tabla 4).

Tabla 4. Backtesting: modelos que no se rechazan

\begin{tabular}{|c|c|c|c|c|c|c|}
\hline \multirow{2}{*}{ Acciones } & \multicolumn{5}{|c|}{ VaR paramétrico } & \multirow[b]{2}{*}{$\begin{array}{c}\text { VaR no } \\
\text { paramétrico }\end{array}$} \\
\hline & & & Modelos & $\begin{array}{c}\mathrm{VaR} \\
\text { paramétrico }\end{array}$ & $\begin{array}{l}\text { Simulación } \\
\text { Montecarlo }\end{array}$ & \\
\hline \multirow{3}{*}{ PFBCOLOMBIA } & \multirow{3}{*}{$\begin{array}{l}\text { GARCH; } \\
\text { T-Student }\end{array}$} & P en días & 54 & 54 & 500 & 54 \\
\hline & & M no P & 49 & 48 & 519 & 53 \\
\hline & & $\begin{array}{c}\text { PROB }(M / N>P) \\
(\%)\end{array}$ & 71,71 & 76,37 & 18,51 & 50,28 \\
\hline \multirow{3}{*}{ ECOPETROL } & \multirow{3}{*}{$\begin{array}{l}\text { GARCH; } \\
\text { T-Student }\end{array}$} & P en días & 54 & 54 & 500 & 54 \\
\hline & & $M$ no $P$ & 27 & 50 & 50 & 53 \\
\hline & & PROB $(M / N>P)(\%)$ & 100,00 & 66,68 & 100,00 & 50,28 \\
\hline
\end{tabular}


María Ines Barbosa Camargo, Alejandra Salazar Sarmiento y Kelly Jhohana Peñaloza Gómez

VaR paramétrico

Acciones

Modelos VaR Simulación VaR no paramétrico Montecarlo paramétrico

\begin{tabular}{|c|c|c|c|c|c|c|}
\hline \multirow{3}{*}{ ETB } & \multirow{3}{*}{$\begin{array}{l}\text { GARCH; } \\
\text { T-Student }\end{array}$} & P en días & 54 & 54 & 500 & 54 \\
\hline & & $M$ no $P$ & 2 & 32 & 31 & 51 \\
\hline & & PROB (M/N>P) (\%) & 100,00 & 99,92 & 100,00 & 61,36 \\
\hline \multirow{3}{*}{ BVC } & \multirow{3}{*}{$\begin{array}{l}\text { GARCH; } \\
\text { T-Student }\end{array}$} & P en días & 54 & 54 & 500 & 54 \\
\hline & & M no $P$ & 18 & 55 & 62 & 53 \\
\hline & & PROB (M/N>P) (\%) & 100,00 & 39,30 & 100,00 & 50,28 \\
\hline \multirow{3}{*}{ CONCONCRET } & \multirow{3}{*}{ TGARCH; GED } & P en días & 54 & 54 & 500 & 54 \\
\hline & & M no $P$ & 22 & 47 & 31 & 53 \\
\hline & & PROB (M/N>P) (\%) & 100,00 & 80,60 & 100,00 & 50,28 \\
\hline \multirow{3}{*}{ BOGOTA } & \multirow{3}{*}{$\begin{array}{l}\text { E-GARCH; } \\
\text { T-Student }\end{array}$} & P en días & 54 & 54 & 500 & 54 \\
\hline & & M no $P$ & 10 & 51 & 114 & 53 \\
\hline & & PROB (M/N>P) (\%) & 100,00 & 61,36 & 100,00 & 50,28 \\
\hline \multirow{3}{*}{ NUTRESA } & \multirow{3}{*}{ GARCH; GED } & P en días & 54 & 54 & 500 & 54 \\
\hline & & M no $P$ & 0 & 51 & 50 & 53 \\
\hline & & PROB (M/N >P) (\%) & 100,00 & 61,36 & 100,00 & 50,28 \\
\hline \multirow{3}{*}{ PFAVAL } & \multirow{3}{*}{$\begin{array}{l}\text { GARCH; } \\
\text { T-Student }\end{array}$} & P en días & 54 & 54 & 500 & 54 \\
\hline & & M no $P$ & 31 & 47 & 47 & 53 \\
\hline & & PROB (M/N>P) (\%) & 99,96 & 80,60 & 100,00 & 50,28 \\
\hline \multirow{3}{*}{ CLH } & \multirow{3}{*}{ GARCH; GED } & P en días & 54 & 54 & 500 & 54 \\
\hline & & M no $P$ & 1 & 56 & 55 & 290 \\
\hline & & PROB $(\mathrm{M} / \mathrm{N}>\mathrm{P})(\%)$ & 100,00 & 34,10 & 100,00 & 0,00 \\
\hline
\end{tabular}

Fuente: elaboración propia

Como resultado del backtesting tenemos que en el VaR no paramétrico el único valor que se rechaza es el de CLH. Por el contrario, en el VaR paramétrico -tanto de distribución normal como de simulación Montecarlo- no se rechaza ningún modelo. Finalmente, de la totalidad de modelos econométricos estimados se rechazan 15 debido a que no logran ajustar la volatilidad, lo que implica que dichos modelos 
subestiman el VaR. Este hallazgo refuerza la importancia de realizar análisis de gestión de riesgo mediante la incorporación de la mayor cantidad de propiedades de las series financieras recogidas, por ejemplo con la posibilidad de combinar técnicas y modelos. En este sentido, el uso de simulación Montecarlo resulta ser el método paramétrico más adecuado en cuanto su implementación considera el mejor ajuste entre varias distribuciones y simulaciones.

\section{CONCLUSIONES}

Este documento investigó los comportamientos de cola de las distribuciones de innovación para las veinticuatro principales acciones en Colombia. Para ello, se estimaron modelos ARMA que buscaban explicar los componentes de la media de los retornos, así como modelos de heterocedasticidad condicionada que incorporaran elementos propios de las series financieras. A pesar de que el modelo T-Student tipo GARCH es capaz de capturar la propiedad de cola pesada en la mayoría de los activos, no tuvo en cuenta el comportamiento asimétrico en dos de los casos, en los que resulta necesario la inclusión de modelos E-GARCH y TARCH.

Si bien en la gestión de riesgo el VaR sigue siendo la medida más utilizada por los profesionales, ya que al implementarla periódicamente y tener una metodología estructurada se pueden evitar desastres financieros, aún es difícil responder a la pregunta que ha suscitado un gran interés en la literatura financiera y que se refiere a cuál es el modelo más apropiado para pronosticar la volatilidad de los rendimientos de los activos, principalmente en mercados financieros emergentes.

Los resultados hallados para las principales acciones del mercado colombiano corroboran la dificultad de elegir un único modelo para el cálculo del VaR, pero dan luces acerca de la necesidad de incorporar en las estimaciones características propias de las series financieras, tales como volatilidad, asimetría, apalancamiento, entre otras. Esto solo se puede obtener por medio del uso de modelos econométricos o simulaciones particulares que diferencien entre activos y también entre distribuciones. Añádase a esto la validez del uso del método paramétrico de distribución normal, ampliamente utilizado de manera empírica.

Una vez calculado el VaR mediante las diferentes metodologías se evidencia que la simulación Montecarlo es la más acertada para valorar el riesgo accionario colombiano, debido a que sus resultados provienen de la incorporación de pruebas de bondad de ajuste que permiten seleccionar entre diversas distribuciones conocidas y frente a una amplia cantidad de repeticiones. El uso de econometría es igualmente válido en la medida en que se realice una comparación entre modelos que conlleve elegir el mejor, es decir aquel que efectivamente recoja las características de las series. 
Finalmente, a partir de los resultados encontrados se llega a la conclusión de que al incorporar más información, el cálculo del VaR es más acertado, por lo que resulta fundamental realizar su cálculo con las diferentes metodologías de análisis existentes para obtener un panorama más completo y de esta manera mejorar la toma de decisiones de inversión.

\section{REFERENCIAS}

Abounoori, Esmaiel, Elmi, Zahra, y Nademi, Younes. (2016). Forecasting Tehran stock Exchange volatility; Markow switching GARCH approach, En: Physica A, N. 445, p. 264-282. Doi: 10.1016/j.physa.2015.10.024

Aloui, Chaker y Mabrouk, Samir. (2010). Value-at-risk estimations of energy commodities via long-memory, asymmetry and fat-tailed GARCH models. En: Energy Policy, Nº. 38, p. 2326-2339. Doi:10.1016/j.enpol.2009.12.020

Andersen, Torben, Benzoni, Luca y Lund, Jesper. (2002). An empirical investigation of continuous-time equity return models. En: Journal of Finance, Vol. 57, №. 3, p. 1239-1284. Doi: $10.1111 / 1540-6261.00460$

Angelidis, Timotheos, Benos, Alexandros y Degiannakis, Stavros. (2004). The use of GARCH models in VaR estimation. En: Statistical Methodology, N. 1, p. 105-128.

Assaf, Ata. (2015). Value-at-Risk analysis in the MENA equity markets: Fat tails and conditional asymmetries in return distributions. En: Journal of Multinational Financial Management, $N^{\circ}$. 29, p. 30-45. Doi: 10.1016/j.mulfin. $\overline{2014} \cdot 11.002$

Bayer, Sebastian. (2018). Combining Value-at-Risk forecasts using penalized quantile Regressions, En: Econometrics and Statistics, N. 8, p. 56-77.

Bentes, Sónia. (2014). Measuring persistence in stock market volatility using the FIGARCH approach. En: Physica A, N. 408, p. 190-197. Doi: 10.1016/j.physa.2014.04.032

Bollerslev, Tim. (1986). Generalized autoregressive conditional heteroskedasticity. En: Journal of Econometrics, N. 31, p. 307-327. Doi: 10.1016/0304-4076(86)90063 1

Bollerslev, T. (1987). A conditionally heteroscedastic time series model for speculative prices and rates of return. En: Review of Economics and Statistics, Nº. 69, p. 542-547.

Castaño, Elkin,. Gómez, Karoll y Gallón, Santiago (2008). Pronóstico y estructuras de volatilidad multiperíodo de la tasa de cambio del peso colombiano. En: Cuadernos de Economía, Vol. XXVII, No. 48, p. 241-266

Cheong, Chin Wen. (2008). Heavy-tailed value-at-risk analysis for Malaysian stock exchange. En: Physica A, No. 387, p. 4285-4298. Doi: 10.1016/j.physa.2008.01.075

Cheong, Chin Wen. (2009). Modeling and forecasting crude oil markets using ARCH-type models. En: Energy Policy, No. 37, p. 2346-2355. Doi: 10.1016/j.enpol.2009.02.026

Chiu, Yen-Chen y Chuang, I-Yuan. (2016). The performance of the switching forecast model of value-at-risk in the Asian stock markets. En: Finance Research Letters, Nº. 18, p. 43-51. Doi: 10.1016/j.frl.2016.03.019 
Comité de Supervisión Bancaria de Basilea (BCBS) (2019). Minimum capital requirements for market risk. Suiza: BCBS.

Degiannakis, Stavros y Potamia, Artemis. (2017). Multiple-days-ahead value-at-risk and expected shortfall forecasting for stock indices, commodities and exchange rates: Inter-day versus intra-day data. En: International Review of Financial Analysis, $N^{\circ}: 49$, p. 176 190. Doi: 10.1016/j.irfa.2016.10.008

Delgado, Luis David y Durango, María Patricia (2018). Estructuración de un portafolio de inversiones con acciones colombianas, En: Semestre Económico, Vol. 21, N․ 46, p. 167-183. Doi: 10.22395/seec.v21n46a7.

Diamandis, Panayiotis, Drakos, Anastassios, Kouretas, Georgios y Zarangas, Leonidas. (2011). Value-at-risk for long and short trading positions: Evidence from developed and emerging equity markets. En: International Review of Financial Analysis, №. 20, p. 165-176. Doi: 10.1016/j.irfa.2011.02.009

Dimitrakopoulos, Dimitris, Kavussanos, Manolis y Spyrou, Spyros. (2010). Value at risk models for volatile emerging markets equity portfolios. En: The Quarterly Review of Economics and Finance, $N^{\circ}$. 50, p. 515-526. Doi: 10.1016/j.qref. 2010.06 .006

Dowd, Kevin y Blake, David. (2006). After Var: the theory, estimation and insurance applications of quantile-based risk measures. En: The Journal of Risk and Insurance, Vol. 73, No. 2, p. 193-229. Doi: 10.1111/j.1539-6975.2006.00171.x

Engle, Robert. (1982). Autoregressive conditional heteroscedasticity with estimates of the variance of United Kingdom inflation. En: Econométrica, Vol. 50, Nº. 4, p. 987-1007.

Ewing, Bradley y Malik, Farroq. (2017). Modelling asymmetric volatility in oil prices under structural breaks. En: Energy Economics, Nº.63, p. 227-233. Doi: 10.1016/j.eneco.2017.03.001

Fan, Ying, Zhang, Yue-Jung, Tsai, Hsien-Tang, y Wei, Yi-Ming. (2008). Estimating 'Value at Risk' of crude oil price and its spillover effect using the GED-GARCH approach. En: Energy Economics, N. 30, p. 3156-3171 Doi: 10.1016/j.eneco.2008.04.002

Gallant, Ronald, Hsieh, David y Tauchen, George. (1997). Estimation of stochastic volatility models with diagnostics. En: Journal of Econometrics, N. 81, p. 159-192.

Giot, Pierre y Laurent, Sébastien. (2004). Modelling daily Value-at-Risk using realized volatility and ARCH type models. En: Journal of Empirical Finance, Nº. 11, p. 379-398. Doi: 10.1016/j. jempfin.2003.04.003

Gonzaléz-Rivera, Gloria, Lee, Tae-Hwy, y Mishra, Santosh. (2004). Forecasting volatility: A reality check based on option pricing, utility function, value-at-risk, and predictive likelihood. En: International Journal of Forecasting, $N^{\circ}$. 20, p.629- 645. Doi: 10.1016/j.ijforecast.2003.10.003

Hartz, Christoph, Mittnik, Stefan, y Paolella, Marc. (2006). Accurate value-at-risk forecasting based on the normal-GARCH model, En: Computational Statistics and Data Analysis, $\mathrm{N}^{\circ}$. 51, p. 2295-2312.

Hull, John, White, Alan. (1998). Incorporating volatility updating into the historical simulation method for VaR. En: The Journal of Risk, N. 1, p. 5-19. 
Jorion, P. (2000). Valor en Riesgo: El nuevo paradigma para el control de riesgos con derivados. México, D.F.: Limusa.

Kupiec, Paul. (1995). Techniques for verifying the accuracy of risk measurement models. En: The Journal of Derivatives, Vol. 3, N. 2, p. 73-84. Doi: 10.3905/jod.1995.407942.

Kuri, K., Ojeda, C., y Ovalle, D. (2015). Modelación de la serie de retornos diarios de la acción de ecopetrol en el periodo: 27/noviembre/2007-25/noviembre/2013. En: Heurística, N. 17, p. $51-57$.

Laporta, Alessandro, Merlo, Luca y Petrella, Lea. (2018). Selection of Value at Risk Models for Energy Commodities, En: Energy Economics, №. 74, p.628-643. Doi: 10.1016/j.eneco.2018.07.009

Mabrouk, Samir. (2016). Forecasting daily conditional volatility and h-step-ahead short and long Value-at-Risk accuracy: Evidence from financial data. En:The Journal of Finance and Data Science, $N^{\circ}$. 2, p. 136-151. Doi: 10.1016/j.jfds.2016.06.001

Mabrouk, Samir y Saadi, Samir. (2012). Parametric Value-at-Risk analysis: Evidence from stock indices. En: The Quarterly Review of Economics and Finance, N ${ }^{\circ}$. 52, p. 305-321. Doi: 10.1016/j.qref.2012.04.006

Markovitz, Harry. (1952). Portfolio selection. En: The Journal of Finance, Vol. 7, Nº. 1, p. 77-91.

Mendoza, Alfonso y Galvanovskis, Evalds (2014). La cópula GED bivariada. Una aplicación en entornos de crisis. En: El Trimestre Económico, Vol. 81, №. 323, p.721-746.

Nelson, Daniel. (1991). Conditional heteroscedasticity in asset returns: a new approach, En: Econometrica, $N^{\circ} .59$, p. 347-370.

Ospina D’Aleman, Federico y Giraldo Sánchez, David. (2009). Aplicación de los modelos GARCH a la estimacion del VaR de acciones colombianas. En: Soluciones de Postgrado EIA, $N^{\circ} 3$. p. 11-24.

Pérez, Fredy y Fernández, Horacio. (2006). Análisis de la volatilidad del índice general de la bolsa de valores de Colombia utilizando modelos $\mathrm{ARCH}$, En: Revista Ingenierías Universidad de Medellín, Vol. 5, N. 8, p. 13-33.

Pino, Anderson, Uribe, Jorge Mario, y Jiménez, Diana. (2017). Relevancia de los inversionistas institucionales en el mercado accionario colombiano. Semestre Económico, Vol. 20, n. 44, p. 45-65. Doi: 10.22395/seec.v20n44a3.

Reboredo, Juan, Rivera-Castro, Miguel y Ugolini, Andrea. (2016). Downside and upside risk spillovers between exchange rates and stock prices. En: Journal of Banking $\mathcal{E}$ Finance, $\mathrm{N}^{\circ}$. 62, p.76-96.

Rivera, David. (2009). Modelación del efecto del día de la semana para los índices accionarios de Colombia mediante un modelo STAR GARCH. En: Revista de Economía del Rosario, Vol. $12 \mathrm{~N}^{\circ}$. 1, p. 1-24.

Sadegui, Mehdi y Shavvalpour, Saeed. (2006). Energy risk management and value at risk modeling, En: Energy Policy, N. 34, p. 3367-3373. 
Valoración de riesgo mediante modelos GARCH y simulación Montecarlo: evidencia del mercado accionario colombiano

Sadorsky, Perry. (2014). Modeling volatility and correlations between emerging market stock prices and the prices of copper, oil and wheat. En: Energy Economics, N. 43, p. 72-81. Doi: 10.1016/j.eneco.2014.02.014

Schaeffer, Roberto, Borba, Bruno, Rathmann, Régis, Szklo, Alexandre y Castelo-Branco, David. (2012). Dow Jones sustainability index transmission to oil stock market returns: A GARCH approach, En: Energy, №.45, p.933-943.

Slim, Skander, Koubaa, Yosra y BenSaida, Ahmed. (2017). Value-at-Risk under Lévy GARCH models: Evidence from global stock markets. En: Journal of International Financial Markets, Institutions \& Money, $\mathrm{N}^{\circ}$. 46, p. 30-53. Doi: 10.1016/j.intfin.2016.08.008

So, Mike y Yu, Philip. (2006). Empirical analysis of GARCH models in value at risk estimation. International. En: Financial Markets, Institutions \& Money, №. 16, p. 180-197.

Su, Ender y Knowles, Thomas. (2006). Asian Pacific stock market volatility modeling and value at risk analysis. En: Emerging Markets Finance and Trade, N. 42, p. 18-62.

Su, Jung Bin. (2015). Value-at-risk estimates of the stock indices in developed and emerging markets including the spillover effects of currency market. En: Economic Modelling, $N^{\circ} .46$, p. 204-224. Doi: 10.1016/j.econmod.2014.12.022

Su, Jung-Bin y Hung, Jui-Cheng. (2011). Empirical analysis of jump dynamics, heavy-tails and skewness on value-at-risk estimation. En: Economic Modelling, №. 28, p. 1117-1130.

Su, Jung-Bin, Lee, Ming-Chih, y Chiu, Chien-Liang (2014). Why does skewness and the fat-tail effect influence value-at-risk estimates? Evidence from alternative capital markets. En: International Review of Economics and Finance, $N^{\circ} .31,59-85$. Doi: 10.1016/j.iref.2013.12.001

Tang, Ta Lun y Shieh, Shwu-Jane. (2006). Long memory in stock index futures markets: A value-at-risk approach. En: Physica A, N: 366, p. 437-448. Doi: 10.1016/j.physa.2005.10.017

Uribe, Jorge Mario. (2007). Caracterización del mercado accionario colombiano, 2001-2006: un análisis comparativo. En: Borradores de Economía, N. 456, p. 1-35.

Wong, Zhen Yao, Chin, Wen Cheon y Tan, Siow Hooi. (2016). Daily value-at-risk modeling and forecast evaluation: The realized volatility approach. En: The Journal of Finance and Data Science, Vol. 2, N. 3, p.171-187.

Yang, Yung-Lieh y Chang, Chia-Lin. (2008). A double-threshold GARCH model of stock market and currency shocks on stock returns, En: Mathematics and Computers in Simulation, $N^{\circ} .79$, p. $458-474$.

Youssef, Manel, Belkacem, Lotfi y Mokni, Khaled. (2015). Value-at-Risk estimation of energy commodities: A long-memory GARCH-EVT approach. En: Energy Economics, No. p. 51, 99-110. Doi: 10.1016/j.eneco.2015.06.010

Zakoian, Jean Michel. (1994). Threshold heteroskedastic models, En: Journal of Economic Dyna mics and Control, Vol. 18, N. 5, p. 931-955. Doi: 10.1016/0165-1889(94)90039-6 